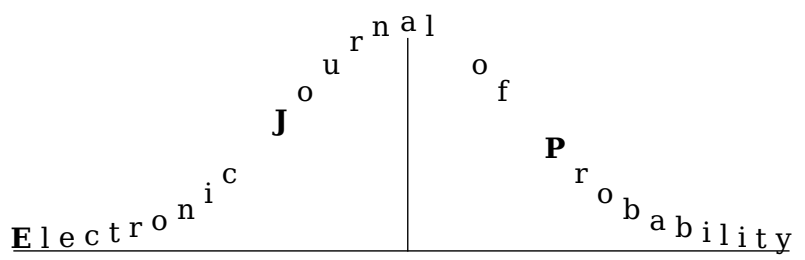

Electron. J. Probab. 27 (2022), article no. 21, 1-17.

ISSN: 1083-6489 https://doi.org/10.1214/22-EJP750

\title{
Analytic proof of multivariate stable local large deviations and application to deterministic dynamical systems*
}

\author{
Ian Melbourne $^{\dagger} \quad$ Dalia Terhesiu ${ }^{\ddagger}$
}

\begin{abstract}
We give a short analytic proof of local large deviations for i.i.d. random variables in the domain of a multivariate $\alpha$-stable law, $\alpha \in(0,1) \cup(1,2]$. Our method simultaneously covers lattice and nonlattice distributions (and mixtures thereof), bypassing aperiodicity considerations. The proof applies also to the dynamical setting.
\end{abstract}

Keywords: local large deviations; multivariate stable laws.

MSC2020 subject classifications: 60F10; 37D20; 37A50.

Submitted to EJP on September 11, 2020, final version accepted on January 30, 2022.

\section{Introduction}

Local large deviation results for i.i.d. random variables in the domain of a stable law have been recently obtained by Caravenna and Doney [7, Theorem 1.1] and refined by Berger [5, Theorem 2.3]. We refer to such results as stable local large deviations (stable LLD).

The aim of this paper is three-fold. First, we provide a new proof of the stable LLD in Theorem 1.1 (we exclude the case $\alpha=1$ but include the case $\alpha=2$ which was previously omitted). Second, in Theorem 1.6, we generalise to the multivariate case which for the main part had also been previously omitted. Our methods bypass aperiodicity considerations and cover lattice and nonlattice distributions simultaneously. Instead of using Fuk-Nagaev inequalities as was done in [5, 7], we give a short analytic proof using Nagaev-type perturbative arguments together with decay of Fourier coefficients. A major advantage of this approach is that it generalises naturally to the dynamical setting. This is the third main aim of this paper where in Theorem 3.2 we establish the

\footnotetext{
*DT was partially supported by EPSRC grant EP/S019286/1.

${ }^{\dagger}$ Mathematics Institute, University of Warwick, Coventry, CV4 7AL, United Kingdom. E-mail: i.melbourne@ warwick.ac.uk

${ }^{\ddagger}$ Mathematisch Instituut, University of Leiden, Niels Bohrweg 1, 2333 CA Leiden, Netherlands.

E-mail: d.e.terhesiu@math. leidenuniv.nl
} 
stable LLD for sequences of nonindependent random variables arising from observables of deterministic dynamical systems.

We begin by recalling the scalar i.i.d. set up in $[5,7]$. Let $X$ be a random variables with $\mathbb{E} X^{2}=\infty$. We suppose that

$$
\mathbb{P}(X>x)=(p+o(1)) \ell(x) x^{-\alpha}, \quad \mathbb{P}(X \leq-x)=(q+o(1)) \ell(x) x^{-\alpha}
$$

as $x \rightarrow \infty$, where $\alpha \in(0,2], \ell:[0, \infty) \rightarrow(0, \infty)$ is slowly varying and $p, q \geq 0$ with $p+q>0$. Equivalently, $X$ is in the domain of an $\alpha$-stable law $Y_{\alpha}$ (determined by $\alpha, p, q$ ). Namely, there are sequences $a_{n}>0, b_{n} \in \mathbb{R}$, such that

$$
\frac{1}{a_{n}}\left(S_{n}-b_{n}\right) \rightarrow_{d} Y_{\alpha}
$$

where $S_{n}=X_{1}+\cdots+X_{n}$ and the $X_{i}$ are independent copies of $X$.

Without loss of generality, we may suppose that $\ell$ is continuous. Set $\tilde{\ell}=\ell$ for $\alpha \in(0,2)$ and $\tilde{\ell}(x)=1+\int_{1}^{1+x} \frac{\ell(u)}{u} d u$ for $\alpha=2$. Then $a_{n}$ satisfies

$$
\lim _{n \rightarrow \infty} \frac{n \tilde{\ell}\left(a_{n}\right)}{a_{n}^{\alpha}}=1
$$

Also,

$$
b_{n}=\left\{\begin{array}{ll}
0 & \alpha \in(0,1) \\
n \mathbb{E}\left(X 1_{\left\{|X| \leq a_{n}\right\}}\right) & \alpha=1 \\
n \mathbb{E} X & \alpha \in(1,2]
\end{array} .\right.
$$

Stable local large deviations (the main topic of this paper) concerns estimates for $\mathbb{P}\left(S_{n} \in J\right)$ for subsets $J \subset \mathbb{R}$ taking into account the location of $J$ :

Theorem 1.1. Assume (1.1) with $\alpha \in(0,2]$. Then for every $h>0$ there is a constant $C>0$ such that

$$
\mathbb{P}\left(S_{n}-b_{n} \in(x-h, x+h]\right) \leq C \frac{n}{a_{n}} \frac{\tilde{\ell}(|x|)}{1+|x|^{\alpha}} \quad \text { for all } n \geq 1, x \in \mathbb{R} .
$$

In particular, in the lattice case where $X$ is supported on $\mathbb{Z}$, there is a constant $C>0$ such that

$$
\mathbb{P}\left(S_{n}-\left[b_{n}\right]=N\right) \leq C \frac{n}{a_{n}} \frac{\tilde{\ell}(|N|)}{1+|N|^{\alpha}} \quad \text { for all } n \geq 1, N \in \mathbb{Z} .
$$

Remark 1.2. Caravenna and Doney [7, Theorem 1.1] proved Theorem 1.6 for $\alpha \in$ $(0,1) \cup(1,2)$ and (amongst other things) this was extended by Berger [5, Theorem 2.3] to the range $\alpha \in(0,2)$ (focusing on the lattice case). Our analytic proof covers the range $\alpha \in(0,1) \cup(1,2]$ so the combined results cover the range $\alpha \in(0,2]$. Our arguments cover the lattice and nonlattice cases simultaneously. As mentioned before, our main contribution is to provide a new proof which generalises easily to the dynamical setting.

In the range $|x| \ll a_{n}$, the estimate (1.2) follows from the local limit theorems of Gnedenko [11, Chap. 9, Sec. 50] and Stone [17], and in particular the estimate is sharp in the range $|x| \approx a_{n}$. Hence the main content of Theorem 1.1 is when $|x| \gg a_{n}$.

Remark 1.3. We have excluded the problematic case $\alpha=1$ which was completely solved by Berger [5]. In fact, our methods apply without modification for $\alpha=1$ in the symmetric case $b_{n}=0$. However, in the nonsymmetric case the estimate in Lemma 2.2(i) below fails (see [10, Lemma 5]). Consequently, without refining our methods further we would obtain a suboptimal estimate in Theorem 1.6 for $\alpha=1, b_{n} \neq 0$.

Remark 1.4. The estimates in Theorem 1.6 are proved under assumption (1.1) which is necessary and sufficient for convergence to the stable law $Y_{\alpha}$. For stronger estimates under more restrictive hypotheses, we refer to $[5,9,12]$. 
Next, we generalise to the multivariate situation. Let $\mathbb{S}^{d-1}=\left\{x \in \mathbb{R}^{d}:|x|=1\right\}$ denote the unit sphere in $\mathbb{R}^{d}$. (Throughout, || denotes the Euclidean norm.)

Definition 1.5. An $\mathbb{R}^{d}$-valued random variable $X$ is regularly varying with index $p$ if there exists a Borel probability measure $\sigma$ on $\mathbb{S}^{d-1}$, such that

$$
\lim _{t \rightarrow \infty} \frac{\mathbb{P}(|X|>\lambda t, X /|X| \in A)}{\mathbb{P}(|X|>t)}=\lambda^{p} \sigma(A)
$$

for all $\lambda>0$ and all Borel sets $A \subset \mathbb{S}^{d-1}$ with $\sigma(\partial A)=0$.

For $p=-\alpha<0$, we say that $X$ is nondegenerate if $\int_{\mathbb{S}^{d-1}}|u \cdot \theta|^{\alpha} d \sigma(\theta)>0$ for all $u \in \mathbb{S}^{d-1}$.

Taking $A=\mathbb{S}^{d-1}$, we have that $|X|$ is a scalar regularly varying function. Hence there exists a slowly varying function $\ell:[0, \infty) \rightarrow(0, \infty)$ such that $\mathbb{P}(|X|>t)=t^{p} \ell(t)$.

Let $X$ be an $\mathbb{R}^{d}$-valued random variable with $\mathbb{E}|X|^{2}=\infty$. We suppose that $X$ is nondegenerate and regularly varying with index $-\alpha$ where $\alpha \in(0,1) \cup(1,2]$. We define $\tilde{\ell}$, $a_{n}$ and $b_{n}$ as in the scalar case (again taking $\ell$ to be continuous).

Let $Y_{\alpha}$ denote the $d$-dimensional stable law with spectral measure $\Lambda=\cos \frac{\pi \alpha}{2} \Gamma(1-\alpha) \sigma$ and characteristic function

$$
\mathbb{E}\left(e^{i s \cdot Y_{\alpha}}\right)=\exp \left\{-\int_{\mathbb{S}^{d-1}}|s \cdot \theta|^{\alpha}\left(1-i \operatorname{sgn}(s \cdot \theta) \tan \frac{\pi \alpha}{2}\right) d \Lambda(\theta)\right\}, \quad s \in \mathbb{R}^{d} .
$$

By [16], $X$ is in the domain of attraction of $Y_{\alpha}$. Indeed, $a_{n}^{-1}\left(S_{n}-b_{n}\right) \rightarrow_{d} Y_{\alpha}$ as $n \rightarrow \infty$ where $S_{n}=X_{1}+\cdots+X_{n}$ and the $X_{i}$ are independent copies of $X$.

Let $\Pi_{h}(x)=\prod_{j=1}^{d}\left(x_{j}-h, x_{j}+h\right]$ for $x \in \mathbb{R}^{d}, h>0$.

Theorem 1.6. Let $\alpha \in(0,1) \cup(1,2]$. For every $h>0$, there is a constant $C>0$ such that

$$
\mathbb{P}\left(S_{n}-b_{n} \in \Pi_{h}(x)\right) \leq C \frac{n}{a_{n}^{d}} \frac{\tilde{\ell}(|x|)}{1+|x|^{\alpha}} \quad \text { for all } n \geq 1, x \in \mathbb{R}^{d} .
$$

Theorem 1.1 for $\alpha \neq 1$ is immediate from Theorem 1.6.

Remark 1.7. We emphasize that our method works simultaneously for lattice and nonlattice distributions, bypassing any aperiodicity assumptions. In particular, our result covers distributions that are jointly lattice and nonlattice avoiding the consideration of numerous different cases that arise in the corresponding local limit theorems (see the discussion in [8]).

Moreover, in the dynamical setting of Section 3, local limit theorems would require additional hypotheses beyond the probabilistic ones, and our method avoids such hypotheses.

Remark 1.8. Berger [6] obtains LLD for multivariate stable laws in the case when they are lattice distributed. Furthermore, [6] allows the scalings $a_{n}$ to vary from component to component.

Our analytic proof of Theorem 1.6 is given in Section 2. In Section 3, we show that our proof applies to a class of deterministic dynamical systems. In fact, we prove a stronger operator stable LLD in Theorem 3.2 which yields the desired stable LLD in Corollary 3.3.

Notation We write $a_{n} \ll b_{n}$ if there are constants $C>0, n_{0} \geq 1$ such that $a_{n} \leq C b_{n}$ for all $n \geq n_{0}$. As usual, $a_{n}=o\left(b_{n}\right)$ means that $\lim _{n \rightarrow \infty} a_{n} / b_{n}=0$ and $a_{n} \sim b_{n}$ means that $\lim _{n \rightarrow \infty} a_{n} / b_{n}=1$. 


\section{Stable local large deviations in the i.i.d. set up}

In this section, we provide an analytic proof of Theorem 1.6 establishing local large deviations for i.i.d. random variables in the domain of a multivariate stable law. We abbreviate $\Pi_{h}(0)$ to $\Pi_{h}$.

Fix $\alpha \in(0,1) \cup(1,2]$, and let $X$ be $\mathbb{R}^{d}$-valued with $\mathbb{E}|X|^{2}=\infty$, nondegenerate and regularly varying with index $-\alpha$. Define $a_{n}$ and $\tilde{\ell}$ as in Section 1 . Since we exclude the case $\alpha=1$, we can suppose without loss of generality that $b_{n}=0$. This is automatic for $\alpha \in(0,1)$ while for $\alpha \in(1,2]$ we can replace $X$ by $X-\mathbb{E} X$. In other words, we suppose that $\mathbb{E} X=0$ for $\alpha \in(1,2]$.

\subsection{Technical lemmas}

The proof of the results obtained here exploits classical approaches $[11,13]$ for characteristic functions collected in Lemmas 2.1 and 2.2 below.

For $s \in \mathbb{R}^{d}$, define $\Psi(s)=\mathbb{E}\left(e^{i s \cdot X}\right)$.

Lemma 2.1. There exist constants $\epsilon, c>0$, such that

$$
|\Psi(s)| \leq \exp \left\{-c|s|^{\alpha} \tilde{\ell}(1 /|s|)\right\} \quad \text { for all } s \in \Pi_{3 \epsilon} .
$$

Proof. Since $S_{n}=X_{1}+\cdots+X_{n}$ is a sum of i.i.d. random vectors and $a_{n}^{-1} S_{n} \rightarrow_{d} Y_{\alpha}$, it follows from the Lévy continuity theorem that $\lim _{n \rightarrow \infty} \Psi\left(s / a_{n}\right)^{n}=\mathbb{E}\left(e^{i s \cdot Y_{\alpha}}\right)$ for all $s \in \mathbb{R}^{d}$. The convergence is uniform on compact sets so $\lim _{n \rightarrow \infty} \Psi\left(u / a_{n}\right)^{n}=\mathbb{E}\left(e^{i u \cdot Y_{\alpha}}\right)$ uniformly in $u \in \mathbb{S}^{d-1}$. Hence

$$
\lim _{n \rightarrow \infty}\left|\Psi\left(u / a_{n}\right)\right|^{n}=e^{-k_{u}} \quad \text { uniformly in } u \in \mathbb{S}^{d-1},
$$

where $k_{u}=\int_{\mathbb{S}^{d-1}}|u \cdot \theta|^{\alpha} d \Lambda(\theta)$. By compactness, $k_{u}$ is bounded and hence

$$
\lim _{n \rightarrow \infty} n \log \left|\Psi\left(u / a_{n}\right)\right|=-k_{u} \quad \text { uniformly in } u \in \mathbb{S}^{d-1} \text {. }
$$

Setting $a_{n}^{-1}=t$ and inverting to obtain $n \sim\left(t^{\alpha} \tilde{\ell}(1 / t)\right)^{-1}$ as $n \rightarrow \infty$, we obtain

$$
\lim _{t \rightarrow 0^{+}}\left(t^{\alpha} \tilde{\ell}(1 / t)\right)^{-1} \log |\Psi(t u)|=-k_{u} \quad \text { uniformly in } u \in \mathbb{S}^{d-1} .
$$

(The details of this step are identical to the last five lines of the proof of [1, Lemma 6.4].) By nondegeneracy and compactness, $\min _{u \in \mathbb{S}^{d-1}} k_{u}>0$. Writing $s=t u$,

$$
\log |\Psi(s)| \sim-k_{u}|s|^{\alpha} \tilde{\ell}(1 /|s|) \quad \text { as } s \rightarrow 0 .
$$

Hence there exists $\epsilon>0$ such that $\log |\Psi(s)| \leq-\frac{1}{2} k_{u}|s|^{\alpha} \tilde{\ell}(1 /|s|)$ for all $s \in \Pi_{3 \epsilon}$ and the result follows with $c=\frac{1}{2} \min _{u \in \mathbb{S}^{d-1}} k_{u}$.

Throughout this section we fix $\epsilon$ so that Lemma 2.1 holds. Let $\partial_{j}=\partial / \partial s_{j}$.

Lemma 2.2. Let $M>0$. There exists $C>0$ such that for all $s, h \in \Pi_{M}$,

(i) $|\Psi(s+h)-\Psi(s)| \leq C|h|^{\alpha} \ell(1 /|h|)$ for $\alpha \in(0,1)$.

(ii) $\left|\partial_{j} \Psi(s+h)-\partial_{j} \Psi(s)\right| \leq C|h|^{\alpha-1} \tilde{\ell}(1 /|h|)$ for $\alpha \in(1,2], j=1, \ldots, d$.

(iii) $\left|\partial_{j} \Psi(s)\right| \leq C|s|^{\alpha-1} \tilde{\ell}(1 /|s|)$ for $\alpha \in(1,2], j=1, \ldots, d$.

Proof. (i) For $K>0$, write $\Psi(s+h)-\Psi(s)=A+B$ where

$$
A=\mathbb{E}\left(1_{\{|X|>K\}}\left(e^{i h \cdot X}-1\right) e^{i s \cdot X}\right), \quad B=\mathbb{E}\left(1_{\{|X| \leq K\}}\left(e^{i h \cdot X}-1\right) e^{i s \cdot X}\right) .
$$

Note that

$$
|A| \leq 2 \mathbb{E} 1_{\{|X|>K\}}=2 \mathbb{P}(|X|>K)=2 K^{-\alpha} \ell(K) .
$$


Analytic proof of multivariate stable local large deviations

Next, let $G(x)=\mathbb{P}(|X| \leq x)$ denote the distribution function of $|X|$, so $1-G(x)=x^{-\alpha} \ell(x)$. Then

$$
|B| \leq|h| \mathbb{E}\left(|X| 1_{\{|X| \leq K\}}\right)=|h| \int_{0}^{K} x d G(x) .
$$

Using integration by parts and Karamata's Theorem,

$$
\begin{aligned}
\int_{0}^{K} x d G(x) & =-\int_{0}^{K} x d(1-G(x))=-\left(\left.x(1-G(x))\right|_{0} ^{K}+\int_{0}^{K}(1-G(x)) d x\right. \\
& \leq \int_{0}^{K}(1-G(x)) d x=\int_{0}^{K} x^{-\alpha} \ell(x) d x=(1-\alpha)^{-1} K^{1-\alpha} \ell(K) .
\end{aligned}
$$

Hence $|B| \ll|h| K^{1-\alpha} \ell(K)$. Taking $K \approx 1 /|h|$ yields the desired estimate.

(ii) For $K>0$, write $\partial_{j} \Psi(s+h)-\partial_{j} \Psi(s)=i(A+B)$ where

$$
A=\mathbb{E}\left(1_{\{|X|>K\}} X_{j}\left(e^{i h \cdot X}-1\right) e^{i s \cdot X}\right), \quad B=\mathbb{E}\left(1_{\{|X| \leq K\}} X_{j}\left(e^{i h \cdot X}-1\right) e^{i s \cdot X}\right) .
$$

Using integration by parts and Karamata's Theorem,

$$
\begin{aligned}
|A| & \leq 2 \mathbb{E}\left(|X| 1_{\{|X|>K\}}\right)=2 \int_{K}^{\infty} x d G(x) \\
& =2 K(1-G(K))+2 \int_{K}^{\infty}(1-G(x)) d x=2 K^{-(\alpha-1)} \ell(K)+2 \int_{K}^{\infty} x^{-\alpha} \ell(x) d x \\
& =2\left\{1+(\alpha-1)^{-1}\right\} K^{-(\alpha-1)} \ell(K) \ll K^{-(\alpha-1)} \tilde{\ell}(K),
\end{aligned}
$$

and

$$
\begin{aligned}
|B| \leq|h| \mathbb{E}\left(|X|^{2} 1_{\{|X| \leq K\}}\right) & =|h| \int_{0}^{K} x^{2} d G(x) \leq 2|h| \int_{0}^{K} x(1-G(x)) d x \\
& =2|h| \int_{0}^{K} x^{-(\alpha-1)} \ell(x) d x=2(2-\alpha)^{-1}|h| K^{2-\alpha} \tilde{\ell}(K) .
\end{aligned}
$$

Again we take $K \approx 1 /|h|$.

(iii) Since $\mathbb{E} X=0$, it follows from part (ii) that $\partial_{j} \Psi(s)=\partial_{j} \Psi(s)-\partial_{j} \Psi(0)$ satisfies the desired estimate.

Lemma 2.3. Let $L:(0, \infty) \rightarrow(0, \infty)$ be a continuous slowly varying function. For all $c>0, \beta \geq 0$, there exists $C>0$ such that for all $n \geq 1$,

$$
\int_{\mathbb{R}^{d}}|s|^{\beta} L(1 /|s|) \exp \left\{-n c|s|^{\alpha} \tilde{\ell}(1 /|s|)\right\} d s \leq C \frac{L\left(a_{n}\right)}{a_{n}^{d+\beta}} .
$$

In particular,

$$
\int_{\Pi_{3 \epsilon}}|s|^{\beta} L(1 /|s|)|\Psi(s)|^{n} d s \leq C \frac{L\left(a_{n}\right)}{a_{n}^{d+\beta}} .
$$

Proof. Let $I_{n}=\int_{\mathbb{R}^{d}}|s|^{\beta} L(1 /|s|) \exp \left\{-n c|s|^{\alpha} \tilde{\ell}(1 /|s|)\right\} d s$. Using the change of variables $s=\sigma / a_{n}$, we obtain

$$
I_{n}=\frac{L\left(a_{n}\right)}{a_{n}^{d+\beta}} J_{n} \quad \text { where } \quad J_{n}=\int_{\mathbb{R}^{d}} \frac{L\left(a_{n} /|\sigma|\right)}{L\left(a_{n}\right)}|\sigma|^{\beta} \exp \left\{-c n|\sigma|^{\alpha} a_{n}^{-\alpha} \tilde{\ell}\left(a_{n} /|\sigma|\right)\right\} d \sigma .
$$

By Potter's bounds, for any $\delta \in(0, \alpha)$, there exists $c^{\prime}>0$ such that

$$
J_{n} \ll \int_{|\sigma|>1}|\sigma|^{\beta+\delta} \exp \left\{-c^{\prime} n|\sigma|^{\alpha-\delta} a_{n}^{-\alpha} \tilde{\ell}\left(a_{n}\right)\right\} d \sigma .
$$


Recalling that $n a_{n}^{-\alpha} \tilde{\ell}\left(a_{n}\right) \sim 1$, there exists $c^{\prime \prime}>0$ such that

$$
J_{n} \ll \int_{|\sigma|>1}|\sigma|^{\beta+\delta} e^{-c^{\prime \prime}|\sigma|^{\alpha-\delta}} d \sigma<\infty .
$$

A similar argument deals with the integral on $\{|\sigma| \leq 1\}$.

The final statement follows from Lemma 2.1 .

\subsection{Proof of the stable LLD}

In this subsection, we prove Theorem 1.6. We suppose without loss of generality that $h=1$; the result for smaller cubes is immediate and the result for larger cubes can be obtained by taking unions of smaller cubes.

As in [17], we convolve with a suitable function $\gamma$ with compactly supported Fourier transform $\hat{\gamma}$. Specifically, fix a continuous integrable function $\gamma_{0}: \mathbb{R} \rightarrow[0, \infty)$ with $\gamma_{0} \geq 1$ on $[-2,2]$ such that its Fourier transform $\widehat{\gamma}_{0}$ is real-valued, even and $C^{2}$ with support in $[-\epsilon, \epsilon] \cdot{ }^{1}$ For $s \in \mathbb{R}$, define

$$
r_{0}(s)=\frac{1}{2 \pi} \frac{\sin s}{s} \widehat{\gamma_{0}}(s) .
$$

We note that $r_{0}$ is $C^{2}$ and supported in $[-\epsilon, \epsilon]$. Define $\gamma: \mathbb{R}^{d} \rightarrow[0, \infty)$ and $r: \mathbb{R}^{d} \rightarrow \mathbb{R}$,

$$
\gamma(y)=\gamma_{0}\left(y_{1}\right) \cdots \gamma_{0}\left(y_{d}\right), \quad r(s)=r_{0}\left(s_{1}\right) \cdots r_{0}\left(s_{d}\right) .
$$

Lemma 2.4. For $n \geq 1, x \in \mathbb{R}^{d}$,

$$
\mathbb{P}\left(S_{n} \in \Pi_{1}(x)\right) \leq \int_{\mathbb{R}^{d}} e^{-i s \cdot x} r(s) \Psi(s)^{n} d s .
$$

Proof. By the Fourier inversion formula,

$$
\gamma(y)=\frac{1}{(2 \pi)^{d}} \int_{\Pi_{\epsilon}} e^{i s \cdot y} \hat{\gamma}(s) d s=\frac{1}{(2 \pi)^{d}} \int_{\Pi_{\epsilon}} e^{-i s \cdot y} \hat{\gamma}(s) d s .
$$

Let $F_{n}(x)=\mathbb{P}\left(S_{n} \in \prod_{j=1}^{d}\left(-\infty, x_{j}\right]\right)$. By Fubini,

$$
\begin{aligned}
\int_{\Pi_{1}(x)} \int_{\mathbb{R}^{d}} \gamma\left(y-y^{\prime}\right) d F_{n}\left(y^{\prime}\right) d y & \\
& =\frac{1}{(2 \pi)^{d}} \int_{\Pi_{\epsilon}}\left(\int_{\Pi_{1}(x)} e^{-i s \cdot y} d y\right) \hat{\gamma}(s)\left(\int_{\mathbb{R}^{d}} e^{i s \cdot y^{\prime}} d F_{n}\left(y^{\prime}\right)\right) d s \\
& =2^{d} \int_{\Pi_{\epsilon}} e^{-i s \cdot x} r(s) \Psi(s)^{n} d s .
\end{aligned}
$$

Next, since $\gamma_{0} \geq 0$ and $\left.\gamma_{0}\right|_{[-2,2]} \geq 1$,

$$
\int_{\mathbb{R}^{d}} \gamma\left(y-y^{\prime}\right) d F_{n}\left(y^{\prime}\right) \geq \int_{\Pi_{2}(y)} d F_{n}\left(y^{\prime}\right)=\mathbb{P}\left(S_{n} \in \Pi_{2}(y)\right) .
$$

Hence

$$
\int_{\Pi_{1}(x)} \int_{\mathbb{R}^{d}} \gamma\left(y-y^{\prime}\right) d F_{n}\left(y^{\prime}\right) d y \geq \int_{\Pi_{1}(x)} \mathbb{P}\left(S_{n} \in \Pi_{2}(y)\right) d y \geq 2^{d} \mathbb{P}\left(S_{n} \in \Pi_{1}(x)\right) .
$$

Combining this with (2.2) yields the desired result.

${ }^{1}$ It is easily verified that such a $\gamma_{0}$ exists. Start with an even $C^{\infty}$ function $\widehat{\gamma_{0}}: \mathbb{R} \rightarrow[0, \infty)$ supported in $\left[-\frac{\epsilon}{2}, \frac{\epsilon}{2}\right]$ with inverse Fourier transform $\gamma_{0}$. Then $\gamma_{0}$ is real-valued and $C^{\infty}$. Taking $\widehat{\gamma_{0}} \not \equiv 0$ ensures that $\gamma_{0}(0)=\frac{1}{2 \pi} \int_{-\infty}^{\infty} \widehat{\gamma_{0}}(\xi) d \xi>0$. Replacing $\gamma_{0}(x)$ by $\gamma_{0}(a x)$ with $a$ sufficiently small ensures that $\gamma_{0}>0$ on $[-2,2]$. (Such a scaling shrinks the support of $\widehat{\gamma_{0}}$, so the new $\widehat{\gamma_{0}}$ remains supported in $\left.-\frac{\epsilon}{2}, \frac{\epsilon}{2}\right]$.) Next, replace $\gamma_{0}$ by $c \gamma_{0}$ for $c$ sufficiently large, ensuring that $\gamma_{0} \geq 1$ on $[-2,2]$. Finally, replace $\gamma_{0}$ by $\gamma_{0}^{2}$ and $\widehat{\gamma_{0}}$ by $\widehat{\gamma_{0}} \star \widehat{\gamma_{0}}$ to ensure that $\gamma_{0} \geq 0$. 
Proof of Theorem 1.6. Recall that we have reduced to the case $h=1$. By Lemma 2.4, it suffices to estimate $I_{n, x}=\int_{\mathbb{R}^{d}} e^{-i s \cdot x} r(s) \Psi(s)^{n} d s$. Since $r$ is bounded and supported in $\Pi_{\epsilon}$, it follows from Lemma 2.3 that

$$
\left|I_{n, x}\right| \ll \int_{\Pi_{\epsilon}}|\Psi(s)|^{n} d s \ll a_{n}^{-d} .
$$

If $n \gg\left(1+|x|^{\alpha}\right) / \tilde{\ell}(|x|)$, then $\left|I_{n, x}\right| \ll a_{n}^{-d} \ll \frac{n}{a_{n}^{d}} \frac{\tilde{\ell}(|x|)}{1+|x|^{\alpha}}$ as required. Hence to complete the proof it suffices to consider the case $n \leq c\left(1+|x|^{\alpha}\right) / \tilde{\ell}(|x|)$ for some $c>0$ fixed. In particular, we can suppose that $|x| \geq \pi / \epsilon$, and it suffices to prove that $\left.\left|I_{n, x}\right| \ll \frac{n}{a_{n}^{d}}|\tilde{\ell}| x\right|^{\alpha}$ for $n \leq c^{\prime}|x|^{\alpha} / \tilde{\ell}(|x|)$. Since $a_{n}^{\alpha} / \tilde{\ell}\left(a_{n}\right) \sim n$ and $y \mapsto y^{\alpha} / \tilde{\ell}(y)$ is asymptotically increasing, this last restriction can be written as $a_{n} \leq|x|$.

The case $\alpha \in(0,1)$. We exploit the modulus of continuity of $\Psi$ (see, for instance, [14, Chapter 1]). Note that $I_{n, x}=-\int_{\mathbb{R}^{d}} e^{-i s \cdot x} r(s-h) \Psi(s-h)^{n} d s$, where $h=\pi x /|x|^{2}$. Hence

$$
\left|I_{n, x}\right|=\frac{1}{2}\left|\int_{\mathbb{R}^{d}} e^{-i s \cdot x}\left(r(s) \Psi(s)^{n}-r(s-h) \Psi(s-h)^{n}\right) d s\right| \leq I_{1}+I_{2}
$$

where

$$
I_{1}=\int_{\mathbb{R}^{d}}\left|r(s)-r(s-h)\left\|\left.\Psi(s)\right|^{n} d s, \quad I_{2}=\int_{\mathbb{R}^{d}}\left|r(s-h) \| \Psi(s)^{n}-\Psi(s-h)^{n}\right| d s .\right.\right.
$$

Since $r$ is supported in $\Pi_{\epsilon}$ and $|x| \geq \pi / \epsilon$, the integrands in $I_{1}$ and $I_{2}$ are supported in $\Pi_{2 \epsilon}$. Using also that $r$ is bounded and Lipschitz,

$$
I_{1} \ll|x|^{-1} \int_{\Pi_{2 \epsilon}}|\Psi(s)|^{n} d s, \quad I_{2} \ll \int_{\Pi_{2 \epsilon}}\left|\Psi(s)^{n}-\Psi(s-h)^{n}\right| d s .
$$

By Lemma 2.3, $I_{1} \ll \frac{1}{a_{n}^{d}} \frac{1}{|x|}$.

Next recall the inequality

$$
\left|u^{n}-v^{n}\right| \leq n|u-v|\left(|u|^{n-1}+|v|^{n-1}\right),
$$

which holds for all $u, v \in \mathbb{C}, n \geq 1$. Using this and Lemma 2.2(i),

$$
\left|\Psi(s)^{n}-\Psi(s-h)^{n}\right| \ll n|x|^{-\alpha} \ell(|x|)\left(|\Psi(s)|^{n-1}+|\Psi(s-h)|^{n-1}\right) .
$$

Hence by Lemma 2.3, $I_{2} \ll n|x|^{-\alpha} \ell(|x|) \int_{\Pi_{3 \epsilon}}|\Psi(s)|^{n-1} d s \ll \frac{n}{a_{n}^{d}} \frac{\ell(|x|)}{|x|^{\alpha}}$.

The case $\alpha \in(1,2]$. Choose $j$ so that $\left|x_{j}\right|=\max \left\{\left|x_{1}\right|, \ldots,\left|x_{d}\right|\right\}$. Let $D_{n}=\Psi^{n-1} \partial_{j} \Psi$. Integrating by parts, $I_{n, x}=E_{1}+E_{2}$ where

$$
E_{1}=\frac{1}{i x_{j}} \int_{\mathbb{R}^{d}} e^{-i s \cdot x} \partial_{j} r(s) \Psi(s)^{n} d s, \quad E_{2}=\frac{n}{i x_{j}} \int_{\mathbb{R}^{d}} e^{-i s \cdot x} r(s) D_{n}(s) d s .
$$

Integrating by parts once more, and using that $r$ is $C^{2}$ and supported in $\Pi_{\epsilon}$,

$$
\begin{aligned}
\left|E_{1}\right| & \leq \frac{1}{x_{j}^{2}} \int_{\Pi_{\epsilon}}\left|\partial_{j}^{2} r(s)\right||\Psi(s)|^{n} d s+\frac{n}{x_{j}^{2}} \int_{\Pi_{\epsilon}}\left|\partial_{j} r(s)\right|\left|D_{n}(s)\right| d s \\
& \ll \frac{1}{|x|^{2}} \int_{\Pi_{\epsilon}}|\Psi(s)|^{n} d s+\frac{n}{|x|^{2}} \int_{\Pi_{\epsilon}}|\Psi(s)|^{n-1} d s .
\end{aligned}
$$

(Here, we used also that $\partial_{j} \Psi$ is bounded on $\Pi_{\epsilon}$ by Lemma 2.2(iii).) By Lemma 2.3,

$$
\left|E_{1}\right| \ll \frac{n}{a_{n}^{d}} \frac{1}{|x|^{2}} \ll \frac{n}{a_{n}^{d}} \frac{\tilde{\ell}(|x|)}{|x|^{\alpha}} .
$$


Next, we exploit the modulus of continuity of $r D_{n}$, writing $h=\pi x_{j}^{-1} e_{j}$ (where $e_{j} \in \mathbb{R}^{d}$ is the $j$ 'th unit vector) and

$$
\begin{aligned}
\left|E_{2}\right| & \leq \frac{n}{\left|x_{j}\right|} \int_{\mathbb{R}^{d}}|r(s)-r(s-h)|\left|D_{n}(s)\right| d s+\frac{n}{\left|x_{j}\right|} \int_{\mathbb{R}^{d}}|r(s-h)|\left|D_{n}(s)-D_{n}(s-h)\right| d s \\
& \ll \frac{n}{|x|^{2}} \int_{\Pi_{2 \epsilon}}|\Psi(s)|^{n-1} d s+\frac{n}{|x|} \int_{\Pi_{2 \epsilon}}\left|D_{n}(s)-D_{n}(s-h)\right| d s .
\end{aligned}
$$

Again, $\frac{n}{|x|^{2}} \int_{\Pi_{2 \epsilon}}|\Psi(s)|^{n-1} d s \ll \frac{n}{a_{n}^{d}} \frac{1}{|x|^{2}} \ll \frac{n}{a_{n}^{d}} \frac{\tilde{\ell}(|x|)}{|x|^{\alpha}}$, so it remains to estimate

$$
J=\frac{n}{|x|} \int_{\Pi_{2 \epsilon}}\left|D_{n}(s)-D_{n}(s-h)\right| d s .
$$

Relabel $\{s, s-h\}=\left\{s_{1}, s_{2}\right\}$ where $\left|\Psi\left(s_{1}\right)\right| \leq\left|\Psi\left(s_{2}\right)\right|$. Then $J=J_{1}+J_{2}$ where $J_{i}=\frac{n}{|x|} \int K_{i}$ for $i=1,2$, and

$$
K_{1}=\left|\Psi\left(s_{1}\right)\right|^{n-1}\left|\partial_{j} \Psi\left(s_{1}\right)-\partial_{j} \Psi\left(s_{2}\right)\right|, \quad K_{2}=\left|\Psi\left(s_{1}\right)^{n-1}-\Psi\left(s_{2}\right)^{n-1}\right|\left|\partial_{j} \Psi\left(s_{2}\right)\right| .
$$

By Lemma 2.2(ii),

$$
K_{1} \ll\left|x_{j}\right|^{1-\alpha} \tilde{\ell}\left(\left|x_{j}\right|\right)\left|\Psi\left(s_{1}\right)\right|^{n-1} \ll|x|^{1-\alpha} \tilde{\ell}(|x|)\left|\Psi\left(s_{1}\right)\right|^{n-1} .
$$

Hence by Lemma 2.3,

$$
J_{1} \ll n \frac{\tilde{\ell}(|x|)}{|x|^{\alpha}} \int_{\Pi_{3 \epsilon}}\left|\Psi(s)^{n-1}\right| d s \ll \frac{n}{a_{n}^{d}} \frac{\tilde{\ell}(|x|)}{|x|^{\alpha}} .
$$

Next, by (2.4),

$$
K_{2} \ll n\left|\partial_{j} \Psi\left(s_{2}\right)\right|\left|\Psi\left(s_{1}\right)-\Psi\left(s_{2}\right)\right|\left|\Psi\left(s_{2}\right)\right|^{n-2} .
$$

By the mean value theorem for vector-valued functions, there exists $s^{*}$ between $s_{1}$ and $s_{2}$ such that

$$
K_{2} \ll n\left|x_{j}\right|^{-1}\left|\partial_{j} \Psi\left(s_{2}\right)\right|\left|\partial_{j} \Psi\left(s^{*}\right)\right|\left|\Psi\left(s_{2}\right)\right|^{n-2} \ll n|x|^{-1}\left(K_{3}+K_{4}\right)
$$

where

$$
K_{3}=\left|\partial_{j} \Psi\left(s_{2}\right)\right|\left|\partial_{j} \Psi\left(s_{2}\right)-\partial_{j} \Psi\left(s^{*}\right)\right|\left|\Psi\left(s_{2}\right)\right|^{n-2}, \quad K_{4}=\left|\partial_{j} \Psi\left(s_{2}\right)\right|^{2}\left|\Psi\left(s_{2}\right)\right|^{n-2} .
$$

Correspondingly, we have $J_{2} \ll J_{3}+J_{4}=n^{2}|x|^{-2}\left(\int K_{3}+\int K_{4}\right)$.

By Lemma 2.2(ii),(iii),

$$
K_{3} \ll|x|^{1-\alpha} \tilde{\ell}(|x|)\left|s_{2}\right|^{\alpha-1} \tilde{\ell}\left(1 /\left|s_{2}\right|\right)\left|\Psi\left(s_{2}\right)\right|^{n-2} .
$$

Hence, by Lemma 2.3,

$$
\begin{aligned}
J_{3} & \ll n^{2}|x|^{-(\alpha+1)} \tilde{\ell}(|x|) \int_{\Pi_{3 \epsilon}}|s|^{\alpha-1} \tilde{\ell}(1 /|s|)|\Psi(s)|^{n-2} d s \ll n^{2}|x|^{-(\alpha+1)} \tilde{\ell}(|x|) a_{n}^{-(d+\alpha-1)} \tilde{\ell}\left(a_{n}\right) \\
& \sim n|x|^{-(\alpha+1)} \tilde{\ell}(|x|) a_{n}^{-(d-1)}=\frac{n}{a_{n}^{d}} \frac{\tilde{\ell}(|x|)}{|x|^{\alpha}} \frac{a_{n}}{|x|} \ll \frac{n}{a_{n}^{d}} \frac{\tilde{\ell}(|x|)}{|x|^{\alpha}}
\end{aligned}
$$

where we have used that $a_{n} \leq|x|$.

Finally, by Lemma 2.2(iii), $K_{4} \ll\left|s_{2}\right|^{2(\alpha-1)} \tilde{\ell}\left(1 /\left|s_{2}\right|\right)^{2}\left|\Psi\left(s_{2}\right)\right|^{n-2}$. Hence, by Lemma 2.3,

$$
\begin{aligned}
J_{4} & \ll n^{2}|x|^{-2} \int_{\Pi_{3 \epsilon}}|s|^{2(\alpha-1)} \tilde{\ell}(1 /|s|)^{2}|\Psi(s)|^{n-2} d s \ll n^{2}|x|^{-2} a_{n}^{-d} a_{n}^{-2(\alpha-1)} \tilde{\ell}\left(a_{n}\right)^{2} \\
& \sim n|x|^{-2} a_{n}^{-d} a_{n}^{2-\alpha} \tilde{\ell}\left(a_{n}\right)=\frac{n}{a_{n}^{d}} \frac{\tilde{\ell}(|x|)}{|x|^{\alpha}} \frac{a_{n}^{2-\alpha} \tilde{\ell}\left(a_{n}\right)}{|x|^{2-\alpha} \tilde{\ell}(|x|)} \ll \frac{n}{a_{n}^{d}} \frac{\tilde{\ell}(|x|)}{|x|^{\alpha}} .
\end{aligned}
$$

Combining the estimates for $J_{1}, J_{3}, J_{4}$ we obtain that $J \ll \frac{n}{a_{n}^{d}} \frac{\tilde{\ell}(|x|)}{|x|^{\alpha}}$ completing the proof. 


\section{Stable LLD for dynamical systems}

In this section we show that the previous results can be generalized to a class of deterministic dynamical systems. The dynamical systems considered here are obtained by iterating a measure-preserving map $f: \Lambda \rightarrow \Lambda$ on a probability space $(\Lambda, \mu)$. Starting with an initial condition $z$ distributed according to $\mu$, the process $z, f z, f^{2} z=f(f z), \ldots$ on $\Lambda^{\mathbb{N}}$ is stationary, with distribution $\mu \otimes \delta_{f z} \otimes \delta_{f^{2} z} \otimes \cdots$. Also, if $v: \Lambda \rightarrow \mathbb{R}^{d}$ is a measurable observable, then the $\mathbb{R}^{d}$-valued process $v, v \circ f, v \circ f^{2}, \ldots$ is stationary. The stationary distribution $\mu \otimes \delta_{f z} \otimes \delta_{f^{2} z} \otimes \cdots$ should be compared with the distribution $\mu \otimes \mu \otimes \mu \otimes \cdots$ in the i.i.d. case and explains the word "deterministic": once the initial condition is specified the future dynamics is uniquely specified with no further randomness. This contrasts with standard probabilistic settings, where fresh randomness is typically injected at each time step. This means that techniques from probability theory have to be reinforced with methods from "smooth ergodic theory" with suitable regularity conditions imposed on the map $f$, the measure $\mu$ and the observable $v$.

The abstract setting in this section includes two classes of deterministic dynamical systems: Gibbs-Markov maps with $v$ piecewise Hölder, and more generally AFU maps with $v$ piecewise bounded variation. (The Gauss map $f(z)=z^{-1}-\left[z^{-1}\right], z \in(0,1]$ is a classical example of a Gibbs-Markov map.) Define $v_{n}=\sum_{k=0}^{n-1} v \circ f^{k}$. For $v$ in the domain of an $\alpha$-stable law $Y_{\alpha}, \alpha \in(0,2]$, it is shown in [1,2] that $v_{n}$ suitably normalised converges in distribution to $Y_{\alpha}$. Here, we prove the corresponding local large deviation estimates.

In Subsection 3.1, we state our main results for deterministic dynamical systems in an abstract functional-analytic framework. Subsections 3.2 and 3.3 contain the proof of these results. In Subsections 3.4 and 3.5, we verify that Gibbs-Markov maps and AFU maps are covered by these results.

\subsection{Dynamical systems set up}

Let $f: \Lambda \rightarrow \Lambda$ be a measure-preserving map on a probability space $(\Lambda, \mu)$. Let $v: \Lambda \rightarrow \mathbb{R}^{d}$ be a measurable observation with $\int_{\Lambda}|v|^{2} d \mu=\infty$. We fix $\alpha \in(0,1) \cup(1,2]$ throughout and assume

(H1) $v$ is nondegenerate and regularly varying with index $-\alpha$ as in Definition 1.5.

Define $\ell, \tilde{\ell}$ and $a_{n}$ as in Section 1. As in the i.i.d. case, we set $b_{n}=0$ for $\alpha<1$ and $b_{n}=n \int_{\Lambda} v d \mu$ for $\alpha>1$.

Let $R: L^{1} \rightarrow L^{1}$ be the transfer operator for $f$ defined via the formula $\int_{\Lambda} R \phi \psi d \mu=$ $\int_{\Lambda} \phi \psi \circ f d \mu$. Given $s \in \mathbb{R}^{d}$, define the perturbed operator $R(s): L^{1} \rightarrow L^{1}$ by $R(s) \phi=$ $R\left(e^{i s \cdot v} \phi\right)$.

We assume that there is a Banach space $\mathcal{B} \subset L^{\infty}$ containing constant functions, with norm \|\| satisfying $|\phi|_{\infty} \leq\|\phi\|$ for $\phi \in \mathcal{B}$, such that

(H2) There exist $\epsilon>0, C>0$ such that for all $s, h \in \Pi_{\epsilon}, j=1, \ldots, d$,

(i) $\|R(s)\| \leq C$ for $\alpha \in(0,2]$ and $\left\|\partial_{j} R(s)\right\| \leq C$ for $\alpha \in(1,2]$.

(ii) $\|R(s+\bar{h})-R(s)\| \leq C|h|^{\alpha} \ell(1 /|h|)$ for $\alpha \in(0,1)$ and $\|R(s+h)-R(s)\| \leq C|h|$ for $\alpha \in(1,2]$.

(iii) $\left\|\partial_{j} R(s+h)-\partial_{j} R(s)\right\| \leq C|h|^{\alpha-1} \tilde{\ell}(1 /|h|)$ for $\alpha \in(1,2]$.

Since $R(0)=R$ and $\mathcal{B}$ contains constant functions, 1 is an eigenvalue of $R(0)$. We assume:

(H3) The eigenvalue 1 is simple, and the remainder of the spectrum of $R(0): \mathcal{B} \rightarrow \mathcal{B}$ is contained in a disk of radius less than 1. 
Let $v_{n}=\sum_{k=0}^{n-1} v \circ f^{k}$. Under these hypotheses, we have distributional convergence $\left(v_{n}-b_{n}\right) / a_{n} \rightarrow_{d} Y_{\alpha}$ to the $d$-dimensional $\alpha$-stable law $Y_{\alpha}$ in (1.3) by [1].

Remark 3.1. In $[1,2,15]$, local limit theorems are proved in various situations under additional aperiodicity assumptions. Our results do not require aperiodicity assumptions, so we do not discuss these issues further.

We can now state the main result in the dynamical setting: namely an operator stable LLD.

Theorem 3.2. Let $\alpha \in(0,1) \cup(1,2]$ and assume (H1)-(H3). Then there exists a constant $C>0$ such that

$$
\left|R^{n} 1_{\left\{v_{n}-b_{n} \in \Pi_{1}(x)\right\}}\right|_{\infty} \leq C \frac{n}{a_{n}^{d}} \frac{\tilde{\ell}(|x|)}{1+|x|^{\alpha}} \text { for all } n \geq 1,|x| \in \mathbb{R}^{d} .
$$

A consequence of Theorem 3.2 is the usual stable LLD.

Corollary 3.3. Let $\alpha \in(0,1) \cup(1,2]$ and assume (H1)-(H3). Then for every $h>0$, there exists $C>0$ such that

$$
\left|\int_{\Lambda} \phi \psi \circ f^{n} 1_{\left\{v_{n}-b_{n} \in \Pi_{h}(x)\right\}} d \mu\right| \leq C|\phi|_{\infty}|\psi|_{1} \frac{n}{a_{n}^{d}} \frac{\tilde{\ell}(|x|)}{1+|x|^{\alpha}}
$$

for all $n \geq 1, x \in \mathbb{R}^{d}, \phi \in L^{\infty}, \psi \in L^{1}$.

In particular, taking $\phi=\psi=1$, we obtain that

$$
\mu\left\{v_{n}-b_{n} \in \Pi_{h}(x)\right\} \leq C \frac{n}{a_{n}^{d}} \frac{\tilde{\ell}(|x|)}{1+|x|^{\alpha}} \quad \text { for all } n \geq 1, x \in \mathbb{R}^{d} .
$$

Proof. As in Section 2.2, we can suppose without loss of generality that $h=1$. Now,

$$
\int_{\Lambda} \phi \psi \circ f^{n} 1_{\left\{v_{n}-b_{n} \in \Pi_{1}(x)\right\}} d \mu=\int_{\Lambda} \psi R^{n}\left(1_{\left\{v_{n}-b_{n} \in \Pi_{1}(x)\right\}} \phi\right) d \mu,
$$

so by positivity of the operator $R$,

$$
\begin{aligned}
\mid \int_{\Lambda} \phi \psi \circ f^{n} & \left.1_{\left\{v_{n}-b_{n} \in \Pi_{1}(x)\right\}} d \mu|\leq| R^{n}\left(1_{\left\{v_{n}-b_{n} \in \Pi_{1}(x)\right\}} \phi\right)\right|_{\infty}|\psi|_{1} \\
& \leq\left|R^{n}\left(1_{\left\{v_{n}-b_{n} \in \Pi_{1}(x)\right\}}|\phi|_{\infty}\right)\right|_{\infty}|\psi|_{1}=\left|R^{n} 1_{\left\{v_{n}-b_{n} \in \Pi_{1}(x)\right\}}\right|_{\infty}|\phi|_{\infty}|\psi|_{1} .
\end{aligned}
$$

The result follows by Theorem 3.2.

The proof of Theorem 3.2 takes up the remainder of this section. We suppose from now on that (H1)-(H3) hold. As in Section 2, we can suppose without loss of generality that $b_{n}=0$. Equivalently, for $\alpha \in(1,2]$ we can suppose that $\int_{\Lambda} v d \mu=0$

\subsection{Technical lemmas}

By (H2) and (H3), there exists $\epsilon>0$ and a continuous family $\lambda(s)$ of simple eigenvalues of $R(s)$ for $s \in \Pi_{3 \epsilon}$ with $\lambda(0)=1$. The associated spectral projections $P(s), s \in \Pi_{3 \epsilon}$, form a continuous family of bounded linear operators on $\mathcal{B}$. Moreover, there is a continuous family of linear operators $Q(s)$ on $\mathcal{B}$ and constants $C>0, \delta_{0} \in(0,1)$ such that

$$
\begin{aligned}
& R(s)=\lambda(s) P(s)+Q(s) \text { for } s \in \Pi_{3 \epsilon} . \\
& \left\|Q(s)^{n}\right\| \leq C \delta_{0}^{n} \quad \text { for } s \in \Pi_{3 \epsilon}, n \geq 1 .
\end{aligned}
$$

Hence we can shrink $\epsilon$ so that

$$
\left\|R(s)^{n}\right\| \leq C|\lambda(s)|^{n} \quad \text { for } s \in \Pi_{3 \epsilon}, n \geq 1 .
$$

Let $\zeta(s)=\frac{P(s) 1}{\int P(s) 1 d \mu}$ be the normalized eigenvector corresponding to $\lambda(s)$. 
Analytic proof of multivariate stable local large deviations

Lemma 3.4. There exists $\epsilon>0$ such that the properties of $R(s)$ listed in (H2) are inherited by $P(s), Q(s), \lambda(s)$ and $\zeta(s)$ for all $s, h \in \Pi_{3 \epsilon}$.

Proof. This is a standard consequence of perturbation theory for smooth families of operators.

The next result is the analogue of Lemmas 2.1 and 2.2(iii) for $\lambda(s)$.

Lemma 3.5. There exist constants $\epsilon, c, C>0$ such that the following hold for all $s \in \Pi_{3 \epsilon}$,

(i) $|\lambda(s)| \leq \exp \left\{-c|s|^{\alpha} \tilde{\ell}(1 /|s|)\right\}$ for $\alpha \in(0,1) \cup(1,2]$.

(ii) $\left|\partial_{j} \lambda(s)\right| \leq C|s|^{\alpha-1} \tilde{\ell}(|1 /| s \mid)$ for $\alpha \in(1,2], j=1, \ldots, d$.

Proof. (i) Write

$$
\begin{aligned}
\lambda(s) & =\int R(s) \zeta(s) d \mu=\int R(s) 1 d \mu+\int(R(s)-R(0))(\zeta(s)-\zeta(0)) d \mu \\
& =\int_{\Lambda} e^{i s \cdot v} d \mu+\int_{\Lambda}(R(s)-R(0))(\zeta(s)-\zeta(0)) d \mu=\Psi(s)+V(s) .
\end{aligned}
$$

The estimate for $\Psi$ in Lemma 2.1 is unchanged (since the distribution of $v$ is given by (H1)) so it suffices to verify that the contributions from $V$ are negligible.

For $\alpha \in(0,1)$, we choose $\alpha^{\prime} \in\left(\frac{1}{2} \alpha, \alpha\right)$. Then $\|R(s)-R(0)\| \ll|s|^{\alpha^{\prime}}$ by (H2) and $\|\zeta(s)-\zeta(0)\| \ll|s|^{\alpha^{\prime}}$ by Lemma 3.4. Since $\mathcal{B} \subset L^{\infty}$,

$$
|V(s)| \ll\|R(s)-R(0)\|\|\zeta(s)-\zeta(0)\| \ll|s|^{2 \alpha^{\prime}}=o\left(|s|^{\alpha} \ell(1 /|s|)\right) .
$$

Similarly, $|V(s)| \ll|s|^{2}=o\left(|s|^{\alpha} \tilde{\ell}(1 /|s|)\right)$ when $\alpha \in(1,2]$. This completes the proof of part (i).

(ii) By the formula in part (i), $\partial_{j} \lambda(0)=i \int_{\Lambda} v_{j} d \mu=0$. Hence $\partial_{j} \lambda(s)=\partial_{j} \lambda(s)-\partial_{j} \lambda(0)$ so the estimate follows from Lemma 3.4.

From now on, $\epsilon>0$ is fixed in accordance with the above properties.

Corollary 3.6. Let $L:(0, \infty) \rightarrow(0, \infty)$ be a continuous slowly varying function. For all $c>0, \beta \geq 0$, there exists $C>0$ such that for all $n \geq 1$,

$$
\int_{\Pi_{3 \epsilon}}|s|^{\beta} L(1 /|s|)|\lambda(s)|^{n} d s \leq C \frac{L\left(a_{n}\right)}{a_{n}^{d+\beta}} .
$$

Proof. This follows from Lemmas 2.3 and 3.5(i).

We require the following estimates on the derivatives of $R(s)^{n}$ and $Q(s)^{n}$.

Lemma 3.7. Let $\alpha \in(1,2]$ and fix $\delta_{1} \in\left(\delta_{0}, 1\right)$. Then there exists $C>0$ such that for all $s, s+h \in \Pi_{3 \epsilon}, j=1, \ldots, d$,

$$
\left\|\partial_{j}\left(R(s)^{n}\right)\right\| \leq C n|\lambda(s)|^{n-1} \quad \text { and } \quad\left\|\partial_{j}\left(Q(s+h)^{n}\right)-\partial_{j}\left(Q(s)^{n}\right)\right\| \leq C \delta_{1}^{n}|h|^{\alpha-1} \tilde{\ell}(1 /|h|) .
$$

Proof. We start from $\partial_{j}\left(R(s)^{n}\right)=\sum_{k=0}^{n-1} R(s)^{k} \partial_{j} R(s) R(s)^{n-k-1}$. By (3.3) and (H2)(i)

$$
\left\|\partial_{j}\left(R(s)^{n}\right)\right\| \ll \sum_{k=0}^{n-1}|\lambda(s)|^{k}|\lambda(s)|^{n-k-1}=n|\lambda(s)|^{n-1}
$$


Analytic proof of multivariate stable local large deviations

Next, fix $\delta_{2} \in\left(\delta_{0}, \delta_{1}\right)$. By (3.2) and Lemma 3.4,

$$
\begin{aligned}
\left\|Q(s+h)^{n}-Q(s)^{n}\right\| & \leq \sum_{k=0}^{n-1}\left\|Q(s+h)^{k}\right\|\|Q(s+h)-Q(s)\|\left\|Q(s)^{n-k-1}\right\| \\
& \ll|h| \sum_{k=0}^{n-1} \delta_{0}^{n} \ll \delta_{2}^{n}|h| .
\end{aligned}
$$

Let $k, m \geq 0$ with $k+m=n-1$. Then

$$
\begin{aligned}
\left(Q^{k} \partial_{j} Q Q^{m}\right)(s+h)-\left(Q^{k} \partial_{j} Q Q^{m}\right)(s)= & \left(Q(s+h)^{k}-Q(s)^{k}\right) \partial_{j} Q(s+h) Q(s+h)^{m} \\
& +Q(s)^{k}\left(\partial_{j} Q(s+h)-\partial_{j} Q(s)\right) Q(s+h)^{m} \\
& +Q(s)^{k} \partial_{j} Q(s)\left(Q(s+h)^{m}-Q(s)^{m}\right)
\end{aligned}
$$

so by (3.2), (3.4) and Lemma 3.4,

$\left\|\left(Q^{k} \partial_{j} Q Q^{m}\right)(s+h)-\left(Q^{k} \partial_{j} Q Q^{m}\right)(s)\right\| \ll \delta_{2}^{n-1}|h|+\delta_{2}^{n-1}|h|^{\alpha-1} \tilde{\ell}(1 /|h|) \ll \delta_{2}^{n}|h|^{\alpha-1} \tilde{\ell}(1 /|h|)$.

Substituting into $\partial_{j}\left(Q(s)^{n}\right)=\sum_{k=0}^{n-1}\left(Q^{k} \partial_{j} Q Q^{n-k-1}\right)(s)$ we obtain

$$
\left\|\partial_{j}\left(Q(s+h)^{n}\right)-\left(\partial_{j} Q(s)^{n}\right)\right\| \ll n \delta_{2}^{n}|h|^{\alpha-1} \tilde{\ell}(1 /|h|) \ll \delta_{1}^{n}|h|^{\alpha-1} \tilde{\ell}(1 /|h|)
$$

as required.

Corollary 3.8. (i) Let $\alpha \in(0,1) \cup(1,2]$. There exists $C>0$ such that for all $|h| \leq \epsilon$,

$$
\int_{\Pi_{2 \epsilon}}\left\|R(s)^{k}\right\|\left\|R(s+h)^{m}\right\| d s \leq C a_{n}^{-d} \text { for all } k, m \geq 0, n \geq 1 \text { with } k+m=n .
$$

(ii) Let $\alpha \in(1,2]$. There exists $C>0$ such that

$$
\int_{\Pi_{2 \epsilon}}\left\|\partial_{j}\left(R(s)^{n}\right)\right\| d s \leq C n a_{n}^{-d} \text { for all } n \geq 1, j=1, \ldots, d .
$$

Proof. (i) By (3.3),

$$
\left\|R(s)^{k}\right\|\left\|R(s+h)^{m}\right\| \ll|\lambda(s)|^{k}|\lambda(s+h)|^{m} \ll|\lambda(s)|^{n}+|\lambda(s+h)|^{n} .
$$

Also, by Lemma 3.7, $\left\|\partial_{j}\left(R(s)^{n}\right)\right\| \ll n|\lambda(s)|^{n-1}$. Hence both parts follow from Corollary 3.6,

\subsection{Proof of the operator stable LLD}

In this subsection, we prove Theorem 3.2. Define $r: \mathbb{R} \rightarrow \mathbb{R}$ as in Section 2.2. Recall that $r$ is $C^{2}$, even, and supported in $\Pi_{\epsilon}$.

Lemma 3.9. $1_{\left\{v_{n} \in \Pi_{1}(x)\right\}} \leq \int_{\Pi_{\epsilon}} e^{-i s \cdot x} r(s) e^{i s \cdot v_{n}} d s$ for $n \geq 1, x \in \mathbb{R}^{d}$.

Proof. Define $\gamma: \mathbb{R}^{d} \rightarrow[0, \infty)$ as in Section 2.2. Since $\gamma \geq 0$ and $\left.\gamma\right|_{\Pi_{2}(0)} \geq 1$,

$$
\begin{aligned}
1_{\left\{v_{n} \in \Pi_{1}(x)\right\}} & =\frac{1}{2^{d}} \int_{\Pi_{1}(x)} 1_{\left\{v_{n} \in \Pi_{1}(x)\right\}} d y \\
& \leq \frac{1}{2^{d}} \int_{\Pi_{1}(x)} 1_{\left\{v_{n} \in \Pi_{2}(y)\right\}} d y \leq \frac{1}{2^{d}} \int_{\Pi_{1}(x)} \gamma\left(y-v_{n}\right) d y .
\end{aligned}
$$

Using the Fourier inversion formula (2.1),

$$
1_{\left\{v_{n} \in \Pi_{1}(x)\right\}} \leq \frac{1}{(4 \pi)^{d}} \int_{\Pi_{\epsilon}}\left(\int_{\Pi_{1}(x)} e^{-i s \cdot y} d y\right) \hat{\gamma}(s) e^{i s \cdot v_{n}} d s=\int_{\Pi_{\epsilon}} e^{-i s \cdot x} r(s) e^{i s \cdot v_{n}} d s
$$

by Fubini. 
Analytic proof of multivariate stable local large deviations

Proof of Theorem 3.2. By Lemma 3.9 and positivity of $R$,

$$
R^{n} 1_{\left\{v_{n} \in \Pi_{1}(x)\right\}} \leq \int_{\Pi_{\epsilon}} e^{-i s \cdot x} r(s) R^{n} e^{i s \cdot v_{n}} d s=A_{n, x} 1
$$

where

$$
A_{n, x}=\int_{\Pi_{\epsilon}} e^{-i s \cdot x} r(s) R(s)^{n} d s
$$

Since $1 \in \mathcal{B}$,

$$
\left|R^{n} 1_{\left\{v_{n} \in \Pi_{1}(x)\right\}}\right|_{\infty} \leq\left|A_{n, x} 1\right|_{\infty} \leq\left\|A_{n, x} 1\right\| \leq\left\|A_{n, x}\right\|\|1\| \ll\left\|A_{n, x}\right\| .
$$

Hence it suffices to estimate $\left\|A_{n, x}\right\|$.

Since $r$ is bounded and supported in $\Pi_{\epsilon}$, it follows that $\left\|A_{n, x}\right\| \ll \int_{\Pi_{\epsilon}}\|R(s)\|^{n} d s$. By (3.3) and Corollary 3.6,

$$
\left\|A_{n, x}\right\| \ll \int_{\Pi_{\epsilon}}|\lambda(s)|^{n} d s \ll a_{n}^{-d} .
$$

Hence, for $n \gg\left(1+|x|^{\alpha}\right) / \tilde{\ell}(|x|)$, we obtain the required estimate $\left\|A_{n, x}\right\| \ll \frac{n}{a_{n}^{d}} \frac{\tilde{\ell}(|x|)}{1+|x|^{\alpha}}$.

As in the proof of Theorem 1.6, it remains to prove that $\left\|A_{n, x}\right\| \ll \frac{n}{a_{n}^{d}} \frac{\tilde{\ell}(|x|)}{|x|^{\alpha}}$ for $a_{n} \leq|x|$, $|x| \geq \pi / \epsilon$.

The case $\alpha \in(0,1)$. Let $h=\pi x /|x|^{2}$. The same modulus of continuity argument as in the i.i.d. case (cf. (2.3)) yields $\left\|A_{n, x}\right\| \leq I_{1}+I_{2}$ where

$$
\begin{aligned}
& I_{1}=\int_{\mathbb{R}^{d}}|r(s)-r(s-h)|\left\|R(s)^{n}\right\| d s \ll|x|^{-1} \int_{\Pi_{2 \epsilon}}\left\|R(s)^{n}\right\| d s, \\
& I_{2}=\int_{\mathbb{R}^{d}}|r(s-h)|\left\|R(s)^{n}-R(s-h)^{n}\right\| d s \ll \int_{\Pi_{2 \epsilon}}\left\|R(s)^{n}-R(s-h)^{n}\right\| d s .
\end{aligned}
$$

By Corollary 3.8(i), $I_{1} \ll|x|^{-1} a_{n}^{-d} \ll \frac{n}{a_{n}^{d}} \frac{\ell(|x|)}{|x|^{\alpha}}$.

Next,

$$
\left\|R(s)^{n}-R(s-h)^{n}\right\| \leq \sum_{k=0}^{n-1}\left\|R(s)^{k}\right\|\|R(s)-R(s-h)\|\left\|R(s-h)^{n-k-1}\right\|,
$$

so by (H2)(ii) and Corollary 3.8(i),

$$
I_{2} \ll \frac{\ell(|x|)}{|x|^{\alpha}} \sum_{k=0}^{n-1} \int_{\Pi_{2 \epsilon}}\left\|R(s)^{k}\right\|\left\|R(s-h)^{n-k-1}\right\| d s \ll \frac{n}{a_{n}^{d}} \frac{\ell(|x|)}{|x|^{\alpha}} .
$$

The case $\alpha \in(1,2]$. Choose $j$ so that $\left|x_{j}\right|=\max \left\{\left|x_{1}\right|, \ldots,\left|x_{d}\right|\right\}$. Integrating by parts, $A_{n, x}=E_{1}+E_{2}$ where

$$
E_{1}=\frac{1}{i x_{j}} \int_{\mathbb{R}^{d}} e^{-i s \cdot x} \partial_{j} r(s) R(s)^{n} d s, \quad E_{2}=\frac{1}{i x_{j}} \int_{\mathbb{R}^{d}} e^{-i s \cdot x} r(s) \partial_{j}\left(R(s)^{n}\right) d s .
$$

Integrating by parts once more and using that $r$ is $C^{2}$ and supported in $\Pi_{\epsilon}$,

$$
\begin{aligned}
\left\|E_{1}\right\| & \leq \frac{1}{x_{j}^{2}} \int_{\mathbb{R}^{d}}\left|\partial_{j}^{2} r(s)\right|\left\|R(s)^{n}\right\| d s+\frac{1}{x_{j}^{2}} \int_{\mathbb{R}^{d}}\left|\partial_{j} r(s)\right|\left\|\partial_{j}\left(R(s)^{n}\right)\right\| d s \\
& \ll \frac{1}{x_{j}^{2}} \int_{\Pi_{\epsilon}}\left\|R(s)^{n}\right\| d s+\frac{1}{x_{j}^{2}} \int_{\Pi_{\epsilon}}\left\|\partial_{j}\left(R(s)^{n}\right)\right\| d s .
\end{aligned}
$$


By Corollary 3.8,

$$
\left\|E_{1}\right\| \ll \frac{1}{a_{n}^{d}} \frac{1}{|x|^{2}}+\frac{n}{a_{n}^{d}} \frac{1}{|x|^{2}} \ll \frac{n}{a_{n}^{d}} \frac{\tilde{\ell}(|x|)}{|x|^{\alpha}} .
$$

Next, we exploit the modulus of continuity of $r \partial_{j}\left(R^{n}\right)$, writing $h=\pi x_{j}^{-1} e_{j}$ and

$$
\begin{aligned}
\left\|E_{2}\right\| & \ll \frac{1}{\left|x_{j}\right|} \int_{\mathbb{R}^{d}}|r(s)-r(s-h)|\left\|\partial_{j}\left(R(s)^{n}\right)\right\| d s \\
& +\frac{1}{\left|x_{j}\right|} \int_{\mathbb{R}^{d}}|r(s-h)|\left\|\partial_{j}\left(R(s)^{n}\right)-\partial_{j}\left(R(s-h)^{n}\right)\right\| d s \\
& \ll \frac{1}{|x|^{2}} \int_{\Pi_{2 \epsilon}}\left\|\partial_{j}\left(R(s)^{n}\right)\right\| d s+\frac{1}{|x|} \int_{\Pi_{2 \epsilon}}\left\|\partial_{j}\left(R(s)^{n}\right)-\partial_{j}\left(R(s-h)^{n}\right)\right\| d s .
\end{aligned}
$$

Again $\frac{1}{|x|^{2}} \int_{\Pi_{2 \epsilon}}\left\|\partial_{j}\left(R(s)^{n}\right)\right\| d s \ll \frac{n}{a_{n}^{d}} \frac{1}{|x|^{2}} \ll \frac{n}{a_{n}^{d}} \frac{\tilde{\ell}(|x|)}{|x|^{\alpha}}$ so it remains to estimate

$$
J=\frac{1}{|x|} \int_{\Pi_{2 \epsilon}}\left\|\partial_{j}\left(R(s)^{n}\right)-\partial_{j}\left(R(s-h)^{n}\right)\right\| d s .
$$

By (3.1),

$$
\partial_{j}\left(R(s)^{n}\right)=n \lambda(s)^{n-1} \partial_{j} \lambda(s) P(s)+\lambda(s)^{n} P^{\prime}(s)+\partial_{j}\left(Q(s)^{n}\right) .
$$

Relabel $\{s, s-h\}=\left\{s_{1}, s_{2}\right\}$ where $\left|\lambda\left(s_{1}\right)\right| \leq\left|\lambda\left(s_{2}\right)\right|$. Then $J \leq F_{1}+\cdots+F_{6}$ where

$$
\begin{aligned}
& F_{1}=\frac{n}{|x|} \int_{\Pi_{2 \epsilon}}\left|\lambda\left(s_{1}\right)^{n-1}-\lambda\left(s_{2}\right)^{n-1}\right|\left|\partial_{j} \lambda\left(s_{2}\right)\right|\left\|P\left(s_{2}\right)\right\| d s, \\
& F_{2}=\frac{n}{|x|} \int_{\Pi_{2 \epsilon}}\left|\lambda\left(s_{2}\right)\right|^{n-1}\left|\partial_{j} \lambda\left(s_{1}\right)-\partial_{j} \lambda\left(s_{2}\right)\right|\left\|P\left(s_{2}\right)\right\| d s \\
& F_{3}=\frac{n}{|x|} \int_{\Pi_{2 \epsilon}}\left|\lambda\left(s_{2}\right)\right|^{n-1}\left|\partial_{j} \lambda\left(s_{2}\right)\right|\left\|P\left(s_{1}\right)-P\left(s_{2}\right)\right\| d s \\
& F_{4}=\frac{1}{|x|} \int_{\Pi_{2 \epsilon}}\left|\lambda\left(s_{1}\right)^{n}-\lambda\left(s_{2}\right)^{n}\right|\left\|P^{\prime}\left(s_{2}\right)\right\| d s \\
& F_{5}=\frac{1}{|x|} \int_{\Pi_{2 \epsilon}}\left|\lambda\left(s_{2}\right)\right|^{n}\left\|P^{\prime}\left(s_{1}\right)-P^{\prime}\left(s_{2}\right)\right\| d s \\
& F_{6}=\frac{1}{|x|} \int_{\Pi_{2 \epsilon}}\left\|\partial_{j}\left(Q^{n}\right)\left(s_{1}\right)-\partial_{j}\left(Q^{n}\right)\left(s_{2}\right)\right\| d s .
\end{aligned}
$$

The hardest term $F_{1}$ is estimated in the same way as $J_{2}$ in the proof of Theorem 1.6 so we write the calculation without the justifications:

$$
\begin{aligned}
F_{1} & \ll \frac{n^{2}}{|x|} \int_{\Pi_{2 \epsilon}}\left|\lambda\left(s_{1}\right)-\lambda\left(s_{2}\right)\right|\left|\lambda\left(s_{2}\right)\right|^{n-2}\left|\partial_{j} \lambda\left(s_{2}\right)\right| d s \\
& \ll \frac{n^{2}}{|x|^{2}} \int_{\Pi_{2 \epsilon}}\left|\partial_{j} \lambda\left(s^{*}\right)\right|\left|\partial_{j} \lambda\left(s_{2}\right)\right|\left|\lambda\left(s_{2}\right)\right|^{n-2} d s \\
& \ll \frac{n^{2}}{|x|^{2}} \int_{\Pi_{2 \epsilon}}\left|\partial_{j} \lambda\left(s^{*}\right)-\partial_{j} \lambda\left(s_{2}\right)\right|\left|\partial_{j} \lambda\left(s_{2}\right)\right|\left|\lambda\left(s_{2}\right)\right|^{n-2} d s+\frac{n^{2}}{|x|^{2}} \int_{\Pi_{2 \epsilon}}\left|\partial_{j} \lambda\left(s_{2}\right)\right|^{2}\left|\lambda\left(s_{2}\right)\right|^{n-2} d s \\
& \ll \frac{n^{2} \tilde{\ell}(|x|)}{|x|^{\alpha+1}} \int_{\Pi_{3 \epsilon}}|s|^{\alpha-1} \tilde{\ell}(1 /|s|)|\lambda(s)|^{n-2} d s+\frac{n^{2}}{|x|^{2}} \int_{\Pi_{3 \epsilon}}|s|^{2(\alpha-1)} \tilde{\ell}(1 /|s|)^{2}|\lambda(s)|^{n-2} d s \\
& \ll \frac{n}{a_{n}^{d}} \frac{\tilde{\ell}(|x|)}{|x|^{\alpha}}\left(\frac{a_{n}}{|x|}+\frac{a_{n}^{2-\alpha} \tilde{\ell}\left(a_{n}\right)}{\left.|x|^{2-\alpha}\right)} \ll \frac{n}{a_{n}^{d}} \frac{\tilde{\ell}(|x|)}{|x|^{\alpha}} .\right.
\end{aligned}
$$

This is the only term that requires Lemma 3.5(ii). The terms $F_{2}, \ldots, F_{5}$ require only the rougher estimates in Lemma 3.4 combined with Corollary 3.6 and we obtain

$$
F_{2} \ll \frac{n}{a_{n}^{d}} \frac{\tilde{\ell}(|x|)}{|x|^{\alpha}}, \quad F_{3}, F_{4} \ll \frac{n}{a_{n}^{d}} \frac{1}{|x|^{2}}, \quad F_{5} \ll \frac{1}{a_{n}^{d}} \frac{\tilde{\ell}(|x|)}{|x|^{\alpha}} .
$$


Finally, by Lemma 3.7, $F_{6} \ll \delta_{1}^{n} \frac{\tilde{\ell}(|x|)}{|x|^{\alpha}}$ which ends the proof.

\subsection{Gibbs-Markov maps}

Let $(\Lambda, \mu)$ be a probability space with an at most countable measurable partition $\left\{\Lambda_{k}\right\}$, and let $f: \Lambda \rightarrow \Lambda$ be an ergodic measure-preserving transformation. Define $s\left(z, z^{\prime}\right)$ to be the least integer $n \geq 0$ such that $f^{n} z$ and $f^{n} z^{\prime}$ lie in distinct partition elements. It is assumed that $s\left(z, z^{\prime}\right)=\infty$ if and only if $z=z^{\prime}$; then $d_{\theta}\left(z, z^{\prime}\right)=\theta^{s\left(z, z^{\prime}\right)}$ is a metric for $\theta \in(0,1)$

Let $g=\frac{d \mu}{d \mu \circ f}: \Lambda \rightarrow \mathbb{R}$. We say that $f$ is a Gibbs-Markov map if

- $f \Lambda_{k}$ is a union of partition elements and $\left.f\right|_{\Lambda_{k}}: \Lambda_{k} \rightarrow f \Lambda_{k}$ is a measurable bijection for each $k \geq 1$;

- $\inf _{k} \mu\left(f \Lambda_{k}\right)>0$;

- There are constants $C>0, \theta \in(0,1)$ such that $\left|\log g(z)-\log g\left(z^{\prime}\right)\right| \leq C d_{\theta}\left(z, z^{\prime}\right)$ for all $z, z^{\prime} \in \Lambda_{k}, k \geq 1$.

Standard references for Gibbs-Markov maps include [1, 4].

Given $\phi: \Lambda \rightarrow \mathbb{R}$, let

$$
D_{k} \phi=\sup _{z, z^{\prime} \in \Lambda_{k}, z \neq z^{\prime}}\left|\phi(z)-\phi\left(z^{\prime}\right)\right| / d_{\theta}\left(z, z^{\prime}\right), \quad|\phi|_{\theta}=\sup _{k \geq 1} D_{k} \phi
$$

We define the Banach space $\mathcal{F}_{\theta} \subset L^{\infty}$ to consist of functions $\phi: \Lambda \rightarrow \mathbb{R}$ such that $|\phi|_{\theta}<\infty$ with norm $\|\phi\|_{\theta}=|\phi|_{\infty}+|\phi|_{\theta}<\infty$. For $\phi: \Lambda \rightarrow \mathbb{R}^{d}$, define $|\phi|_{\theta}=\max _{j=1, \ldots, d}\left|\phi_{j}\right|_{\theta}$.

Proposition 3.10. Assume $f$ is a mixing Gibbs-Markov map and let $v: \Lambda \rightarrow \mathbb{R}^{d}$ with $\int_{\Lambda}|v|^{2} d \mu=\infty$ and $|v|_{\theta}<\infty$. Fix $\alpha \in(0,1) \cup(1,2]$ and assume that $v$ satisfies (H1).

Then conditions (H1)-(H3) are satisfied with Banach space $\mathcal{B}=\mathcal{F}_{\theta}$.

Proof. Condition (H1) is satisfied by assumption and condition (H3) is well-known for mixing Gibbs-Markov maps [1, 4]. It remains to verify that (H2) holds. In fact, for any $M>0$ the conditions in (H2) hold for all $|s| \leq M,|h| \leq 1$. We verify this for (H2)(iii). All the other calculations are simpler and hence omitted.

Now $\left(\partial_{j} R(s+h)-\partial_{j} R(s)\right) \phi=i R(\phi \psi)$ where $\psi=v_{j} e^{i s \cdot v}\left(e^{i h \cdot v}-1\right)$. A standard calculation shows that

$$
\|R(\phi \psi)\|_{\theta} \ll \sum_{k} \mu\left(\Lambda_{k}\right)\left(\sup _{k}|\phi \psi|+D_{k}(\phi \psi)\right) \leq\|\phi\|_{\theta} \sum_{k} \mu\left(\Lambda_{k}\right)\left(2 \sup _{k}|\psi|+D_{k} \psi\right) .
$$

where $\sup _{k}=\sup _{\Lambda_{k}}$ and $\inf _{k}=\inf _{\Lambda_{k}}$. Hence

$$
\left\|\partial_{j} R(s+h)-\partial_{j} R(s)\right\|_{\theta} \ll \sum_{k} \mu\left(\Lambda_{k}\right)\left\{\sup _{k}\left|v_{j}\left(e^{i h \cdot v}-1\right)\right|+D_{k}\left(v e^{i s \cdot v}\left(e^{i h \cdot v}-1\right)\right)\right\} .
$$

Also, $D_{k} e^{i s \cdot v} \leq|s||v|_{\theta} \ll|s|$, so $\left\|\partial_{j} R(s+h)-\partial_{j} R(s)\right\|_{\theta} \ll S_{1}+S_{2}+S_{3}+S_{4}$, where

$$
\begin{aligned}
& S_{1}=\sum_{k} \mu\left(\Lambda_{k}\right) \sup _{k}\left|v_{j}\left(e^{i h \cdot v}-1\right)\right|, \\
& S_{2}=\sum_{k} \mu\left(\Lambda_{k}\right) \sup _{k}\left|v_{j}\left(e^{i h \cdot v}-1\right)\right| D_{k} e^{i s \cdot v} \ll|s| S_{1} \leq M S_{1}, \\
& S_{3}=\sum_{k} \mu\left(\Lambda_{k}\right) \sup _{k}|v| D_{k} e^{i h \cdot v} \leq|h| \sum_{k} \mu\left(\Lambda_{k}\right) \sup _{k}|v|, \\
& S_{4}=\sum_{k} \mu\left(\Lambda_{k}\right) \sup _{k}\left|e^{i h \cdot v}-1\right| D_{k} v \ll S_{3} .
\end{aligned}
$$

Next, $\sup _{k}|v|-\inf _{k}|v| \leq|v|_{\theta} \ll 1$. Hence

$$
\sum_{k} \mu\left(\Lambda_{k}\right) \sup _{k}|v| \ll \sum_{k} \mu\left(\Lambda_{k}\right)\left(1+\inf _{k}|v|\right) \leq 1+\int_{\Lambda}|v| d \mu
$$


and we obtain $S_{3}, S_{4} \ll|h|$.

Finally,

$\sup _{k}\left|v_{j}\left(e^{i h \cdot v}-1\right)\right| \ll\left(1+\inf _{k}|v|\right)\left(|h|+\inf _{k}\left|e^{i h \cdot v}-1\right|\right) \ll|h|\left(1+\inf _{k}|v|\right)+\inf _{k}\left|v_{j}\left(e^{i h \cdot v}-1\right)\right|$,

and so

$$
\begin{aligned}
S_{1} & \ll \sum_{k} \mu\left(\Lambda_{k}\right)\left(|h|\left(1+\inf _{k}|v|\right)+\inf _{k}\left|v_{j}\left(e^{i h \cdot v}-1\right)\right|\right) \\
& \leq|h|\left(1+\int_{\Lambda}|v| d \mu\right)+\int_{\Lambda}\left|v_{j}\left(e^{i h \cdot v}-1\right)\right| d \mu \ll|h|+\int_{\Lambda}\left|v_{j}\left(e^{i h \cdot v}-1\right)\right| d \mu .
\end{aligned}
$$

The conditions on $v$ are the same as those on $X$ in Theorem 1.6, so

$$
\int_{\Lambda}\left|v_{j}\left(e^{i h \cdot v}-1\right)\right| d \mu=\mathbb{E}\left|X_{j}\left(e^{i h \cdot X}-1\right)\right| \ll|h|^{\alpha-1} \tilde{\ell}(1 /|h|)
$$

by the proof of Lemma 2.2(ii). Hence $S_{1}, S_{2} \ll|h|+|h|^{\alpha-1} \tilde{\ell}(1 /|h|)$.

Altogether, $\left\|\partial_{j} R(s+h)-\partial_{j} R(s)\right\|_{\theta} \ll|h|+|h|^{\alpha-1} \tilde{\ell}(1 /|h|) \ll|h|^{\alpha-1} \tilde{\ell}(1 /|h|)$ as required.

\subsection{AFU maps}

Let $\Lambda=[0,1]$ with measurable partition $\{I\}$ consisting of open intervals. A map $f: \Lambda \rightarrow \Lambda$ is called AFU if $\left.f\right|_{I}$ is $C^{2}$ and strictly monotone for each $I$, and

(A) (Adler's condition) $f^{\prime \prime} /\left(f^{\prime}\right)^{2}$ is bounded on $\bigcup I$.

(F) (finite images) The set of images $\{f I\}$ is finite.

(U) (uniform expansion) There exists $\rho>1$ such that $\left|f^{\prime}\right| \geq \rho$ on $\bigcup I$.

A standard reference for such maps is [18] (see also [3]). Since AFU maps are not necessarily Markov, the Hölder spaces $\mathcal{F}$ are not preserved by the transfer operator of $f$ and it is standard to consider the space of bounded variation functions. Accordingly, we define the Banach space $\mathcal{B}=\mathrm{BV} \subset L^{\infty}$ to consist of functions $\phi: \Lambda \rightarrow \mathbb{R}$ such that $\operatorname{Var} \phi<\infty$ with norm $\|\phi\|=|\phi|_{\infty}+\operatorname{Var} \phi$. Here

$$
\operatorname{Var} \phi=\sup _{0=z_{0}<\cdots<z_{k}=1} \sum_{i=1}^{k}\left|\phi\left(z_{i}\right)-\phi\left(z_{i-1}\right)\right|
$$

denotes the variation of $\phi$ on $\Lambda$. Also, we let $\operatorname{Var}_{I} \phi$ denote the variation of $\phi$ on $I$. For $\phi: \Lambda \rightarrow \mathbb{R}^{d}$, define $\operatorname{Var} \phi=\max _{j=1, \ldots, d} \operatorname{Var} \phi_{j}$.

We suppose that $f: \Lambda \rightarrow \Lambda$ is topologically mixing. Then there is a unique absolutely continuous $f$-invariant probability measure $\mu$, and $\mu$ is mixing.

Proposition 3.11. Assume $f$ is a topologically mixing AFU map and let $v: \Lambda \rightarrow \mathbb{R}^{d}$ with $\int_{\Lambda}|v|^{2} d \mu=\infty$ and $\sup _{I} \operatorname{Var}_{I} v<\infty$. Fix $\alpha \in(0,1) \cup(1,2]$ and assume that the tails of $v$ satisfy (H1).

Then conditions (H1)-(H3) are satisfied with Banach space $\mathcal{B}=\mathrm{BV}$.

Proof. The proof essentially goes word for word as the proof of Proposition 3.10 with minor changes. Condition (H1) is satisfied by assumption and condition (H3) is wellknown for mixing AFU maps. It remains to verify that (H2) holds. Fix $M>0$. As before, we verify (H2)(iii) for all $|s| \leq M,|h| \leq 1$; the other calculations being simpler.

Again, $\left(\partial_{j} R(s+h)-\partial_{j} R(s)\right) \phi=i R(\phi \psi)$ where $\psi=v_{j} e^{i s \cdot v}\left(e^{i h \cdot v}-1\right)$, and a standard calculation shows that

$$
\|R(\phi \psi)\| \ll\|\phi\| \sum_{I} \mu(I)\left(\sup _{I}|\psi|+\operatorname{Var}_{I} \psi\right) .
$$


Also, $\operatorname{Var}_{I} e^{i s \cdot v} \leq|s| \operatorname{Var}_{I} v \ll|s|$, so $\left\|R^{\prime}(s+h)-R^{\prime}(s)\right\| \leq S_{1}+S_{2}+S_{3}+S_{4}$ where

$$
\begin{aligned}
& S_{1}=\sum_{I} \mu(I) \sup _{I}\left|v_{j}\left(e^{i h \cdot v}-1\right)\right| \\
& S_{2}=\sum_{I} \mu(I) \sup _{I}\left|v_{j}\left(e^{i h \cdot v}-1\right)\right| \operatorname{Var}_{I} e^{i s \cdot v} \ll|s| S_{1} \leq M S_{1}, \\
& S_{3}=\sum_{I} \mu(I) \sup _{I}|v| \operatorname{Var}_{I} e^{i h \cdot v} \leq|h| \sum_{I} \mu(I) \sup _{I}|v|, \\
& S_{4}=\sum_{I} \mu(I) \sup _{I}\left|e^{i h \cdot v}-1\right| \operatorname{Var}_{I} v \ll S_{3} .
\end{aligned}
$$

The calculation continues exactly as in Proposition 3.10 and we omit the remaining details.

\section{References}

[1] J. Aaronson and M. Denker. Local limit theorems for partial sums of stationary sequences generated by Gibbs-Markov maps. Stoch. Dyn. 1 (2001) 193-237. MR1840194

[2] J. Aaronson and M. Denker. A local limit theorem for stationary processes in the domain of attraction of a normal distribution. In N. Balakrishnan, I.A. Ibragimov, V.B. Nevzorov, eds., Asymptotic methods in probability and statistics with applications. International conference, St. Petersburg, Russia, 1998, Basel: Birkhäuser, (2001) 215-224. MR1890313

[3] J. Aaronson, M. Denker, O. Sarig and R. Zweimüller. Aperiodicity of cocycles and conditional local limit theorems. Stoch. Dyn. 4 (2004) 31-62. MR2069366

[4] J. Aaronson, M. Denker and M. Urbański. Ergodic theory for Markov fibred systems and parabolic rational maps. Trans. Amer. Math. Soc. 337 (1993) 495-548. MR1107025

[5] Q. Berger. Notes on random walks in the Cauchy domain of attraction. Probab. Theory Related Fields 175 (2019) 1-44. MR4009704

[6] Q. Berger. Strong renewal theorems and local large deviations for multivariate random walks and renewals. Electron. J. Probab. 24 (2019) Paper No. 46, 47. MR3949271

[7] F. Caravenna and R. A. Doney. Local large deviations and the strong renewal theorem. Electron. J. Probab. 24 (2019) 1-48. MR3978222

[8] R. A. Doney. A bivariate local limit theorem. J. Multivariate Anal. 36 (1991) 95-102. MR1094270

[9] R. A. Doney. One-sided local large deviation and renewal theorems in the case of infinite mean. Probab. Theory Related Fields 107 (1997) 451-465. MR1440141

[10] K. B. Erickson. Strong renewal theorems with infinite mean. Trans. Amer. Math. Soc. 151 (1970) 263-291. MR0268976

[11] B. V. Gnedenko and A. N. Kolmogorov, Limit distributions for sums of independent random variables, Translated from the Russian, annotated, and revised by K. L. Chung. With appendices by J. L. Doob and P. L. Hsu. Revised edition, Addison-Wesley Publishing Co., Reading, Mass.-London-Don Mills., Ont., 1968. MR0233400

[12] S. Gouëzel. Correlation asymptotics from large deviations in dynamical systems with infinite measure. Colloq. Math. 125 (2011) 193-212 MR2871313

[13] I. A. Ibragimov and Y. V. Linnik. Independent and stationary sequences of random variables. Wolters-Noordhoff Publishing, Groningen, 1971. MR0322926

[14] Y. Katznelson. An Introduction to Harmonic Analysis. Dover, New York, 1976. MR0422992

[15] I. Melbourne and D. Terhesiu. Renewal theorems and mixing for non Markov flows with infinite measure. Ann Inst. H. Poincaré (B) Probab. Statist. 56 (2020) 449-476. MR4058995

[16] E. L. Rvačeva. On domains of attraction of multidimensional distributions. L vov. Gos. Univ. Uč. Zap. Ser. Meh.-Mat. 29 (1954) 5-44. MR0076211

[17] C. Stone. A local limit theorem for nonlattice multi-dimensional distribution functions. Ann. Math. Statist. 36 (1965) 546-551. MR0175166

[18] R. Zweimüller. Ergodic structure and invariant densities of non-Markovian interval maps with indifferent fixed points. Nonlinearity 11 (1998) 1263-1276. MR1644385

Acknowledgments. We are grateful to the referees for several helpful comments and suggestions. 


\section{Electronic Journal of Probability Electronic Communications in Probability}

\section{Advantages of publishing in EJP-ECP}

- Very high standards

- Free for authors, free for readers

- Quick publication (no backlog)

- Secure publication $\left(\mathrm{LOCKSS}^{1}\right)$

- Easy interface (EJMS²)

\section{Economical model of EJP-ECP}

- Non profit, sponsored by $\mathrm{IMS}^{3}, \mathrm{BS}^{4}$, ProjectEuclid ${ }^{5}$

- Purely electronic

\section{Help keep the journal free and vigorous}

- Donate to the IMS open access fund ${ }^{6}$ (click here to donate!)

- Submit your best articles to EJP-ECP

- Choose EJP-ECP over for-profit journals

\footnotetext{
${ }^{1}$ LOCKSS: Lots of Copies Keep Stuff Safe http://www. lockss.org/

${ }^{2}$ EJMS: Electronic Journal Management System http://www.vtex.lt/en/ejms.html

${ }^{3}$ IMS: Institute of Mathematical Statistics http://www.imstat.org/

${ }^{4}$ BS: Bernoulli Society http://www. bernoulli-society.org/

${ }^{5}$ Project Euclid: https://projecteuclid.org/

${ }^{6}$ IMS Open Access Fund: http://www.imstat.org/publications/open.htm
} 